\title{
Cardiac Stem Cells Regenerate the Infarcted Heart
}

\author{
J. Kajstura, K. Urbanek, M. Rota, A. Leri and P. Anversa \\ Cardiovascular Research Institute, New York Medical College, Valhalla, New York 10595
}

Cardiac stem and early committed cells (CSCs-ECCs) expressing the stem cell antigens c-kit, MDR1 and Sca-1 have been identified in the heart [1-3]. In the rat myocardium, c-kit ${ }^{\mathrm{POS}}$ cells exhibit all the properties of stem cells [1]. They are self-renewing, clonogenic and multipotent giving rise to myocytes, smooth muscle and endothelial cells in vitro and in vivo. MDR ${ }^{\mathrm{POS}}$ and Sca- ${ }^{\mathrm{POS}}$ cells behave similarly. The presence of CSCs-ECCs throughout the heart raises the question why they fail to respond to ischemic injury with regeneration of myocytes and coronary vessels and restoration of function. For this reason, we explored the possibility that CSCs-ECCs, if properly activated, translocate to areas of damage, multiply and differentiate forming functionally competent myocardium.

Hepatocyte growth factor (HGF) stimulates cell migration [4-6] through induction of metalloproteinases (MMPs) [7] that by breaking down the extracellular matrix favor cell locomotion, homing and tissue reconstitution. The receptor of HGF, c-Met, is expressed in bone marrow progenitor cells (BMPCs), satellite cells and embryonic myocytes [8-10]. IGF-1 is mitogenic, antiapoptotic and necessary for neural stem cell growth $[11,12]$. IGF-1 promotes myocyte formation [13] and attenuates myocyte death after infarction [14]. Together, these findings prompted us to determine whether CSCs-ECCs express c-Met and whether HGF stimulates their migration to the infarcted myocardium. If CSCs-ECCs express IGF-1R, IGF-1 may protect their viability and enhance their growth within the infarct.

Myocardial infarction was produced in mice and HGF and/or IGF-1 were locally injected to trigger migration and homing of CSCs-ECCs to the ischemic tissue. The impact of this intervention on myocardial regeneration, heart function and animal survival was determined acutely, at healing and long thereafter to evaluate its therapeutic efficacy.

Translocation of CSCs-ECCs in the heart can occur via the coronary circulation, the interstitium or both. To identify this path, 2 days after the injection of the EGFP-retrovirus, the coronary circulation was perfused with rhodamine-labeled dextran and the GFs were administrated at the time of observation by two-photon microscopy. The coronary vasculature was recognized by red fluorescence and $\mathrm{EGFP}^{\mathrm{POS}}$ cells by green fluorescence. In all cases, $\mathrm{EGFP}^{\mathrm{POS}}$ cells were located in the interstitium outside the vessels, suggesting that the coronary circulation was not primarily implicated in the translocation of these cells within the myocardium.

The new myocardium was composed of myocytes and coronary vessels that changed in proportion with time. At 16 days, myocytes varied in volume from 600 to $7,200 \mu^{3}$ and, at 4 months, from 700 to $20,000 \mu^{3}$. At both intervals, treated-hearts generated an average 3.2 million myocytes to compensate for an average loss of 2.2 million cells. There were $43 \pm 13$ arterioles and $155 \pm 48$ capillaries $/ \mathrm{mm}^{2}$ new myocardium at 16 days, and $31 \pm 6$ arterioles and $390 \pm 56$ capillaries at 4 
months. For comparison, there are $\sim 10$ arterioles and $\sim 3,500$ capillaries $/ \mathrm{mm}^{2}$ myocardium in the adult mouse heart. New vessels contained erythrocytes.

The discrepancy in size between old and new myocytes makes it unlikely that fusion [1, 15-17] of the activated CSCs with spared myocytes was implicated in the differentiation of these cells into myocytes. Moreover, to rule out fusion of CSCs with other cells, the number of X-chromosomes was evaluated in the regenerated myocytes of the female infarcted mice treated with GFs. At most, two $\mathrm{X}$-chromosomes were detected in the nuclei strongly arguing against cell fusion as a mechanism of myocyte formation. Finally, cycling and non-cycling new and old myocytes were identified by Ki67 and DNA content was measured in individual nuclei. Mouse lymphocytes were used as control. Nuclei of non-cycling myocytes and lymphocytes had 2C DNA content, while Ki67 ${ }^{\mathrm{POS}}$ nuclei had DNA content intermediate between diploid and tetraploid further excluding that cell fusion played an important role in cardiac repair. Myocardial regeneration induced by growth factors increased survival and rescued animals with infarcts up to $86 \%$ of the ventricle, which are commonly incompatible with life.

In conclusion, the local stimulation of resident CSCs-ECCs can be accomplished by minimally invasive procedures. This approach overcomes the complications associated with the systemic administration of cytokines or the injection of a large number of BMPCs, skeletal myoblasts and embryonic stem cells. The development of a simple and effective method to induce stimulation, translocation and differentiation of CSCs-ECCs to the damaged myocardium holds clinical promise and might serve as a model for repair of other tissues.

References

[1] A.P. Beltrami et al. Cell 114 (2003) 763.

[2] H. Oh et al. Proc. Natl. Acad. Sci. USA 100 (2003) 12313.

[3] K. Matsuura et al. J. Biol. Chem. 279 (2004) 11384-11391.

[4] E.M. Powell et al. Neuron. 30, (2001) 79-89.

[5] H. Ma et al. Circ. Res. 28 (2003) 1066.

[6] S. Kermorgant et al. EMBO J. 23 (2004) 3721.

[7] R. Hamasuna et al. Int. J. Cancer 82 (1999) 274.

[8] D.A. Rappolee et al. Circ. Res. 78 (1996) 1028.

[9] I.S. Weimar et al. Exp. Hematol. 26 (1998) 885.

[10] C. Birchmeier and H. Brohmann Curr. Opin. Cell Biol. 12 (2000) 725.

[11] G.J. Brooker et al. J. Neurosci. Res. 59 (2000) 332.

[12] Y. Arsenijevic et al. J. Neurosci. 21 (2001) 7194.

[13] K. Reiss et al. Proc. Natl. Acad. Sci. USA. 93 (1996) 8630.

[14] Q. Li et al. J. Clin. Invest. 100 (1997) 1991.

[15] M. Alvarez-Dolado et al. Nature 425 (2003) 968.

[16] J.M. Weimann et al. Nat. Cell Biol. 5 (2003) 959.

[17] M.A. Rudnicki Nat. Med. 9 (2003) 1461.

[18] This work was supported by grants from the NIH. Retrovirus expressing EGFP was obtained from Drs. D. Williams and R. E. Harris (Children's Hospital; Cincinnati, OH). 